BUTP-9518

hep-ph/9505209

\title{
An exact analytic description of neutrino oscillations in matter with exponentially varying density for arbitrary number of neutrino species
}

\author{
E. Torrente Lujan. Inst. fur Theoretische Physik, Universitat Bern \\ Sidlerstrasse 5, 3012 Bern, Switzerland. \\ e-mail: e.torrente@cern.ch
}

\begin{abstract}
Exact analytical expressions in terms of generalized confluent hypergeometric functions for the transition amplitudes of neutrino oscillations in presence of matter are computed for an arbitrary number of species. The density of matter is assumed to be exponentially decaying. The results can be used for the description of matter-induced neutrino oscillations in the Sun which can take place when the solar neutrinos propagate radially from the interior to the surface. Expressions are particularly simple in the limit of infinite propagation time as is suitable for the case of detection at Earth.

PACS: 14.60.Pq, 02.30.Gp, 02.30.Hq
\end{abstract}




\section{Introduction}

The explanation of the solar neutrino flux problem by maximally mixed neutrino oscillations in vacuum or by resonance-enhanced oscillations without large mixing (MSW effect)

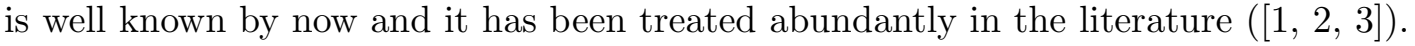

For stable and relativistic neutrinos $\nu=\left(\nu_{i}, i=1, N_{\nu}\right)$ of equal momentum the propagation in vacuum is described in the basis of mass eigenstates by

$$
i \partial_{t} \nu=H^{0} \nu
$$

where

$$
H^{0}=\operatorname{Diag}\left(E_{i}=\sqrt{ }\left(p^{2}+m^{2}\right) \simeq p+m_{i}^{2} / 2 E\right)
$$

The common constant $p$ can be absorbed as an unimportant phase and is usually omitted.

The weak eigenstates $\nu^{\prime}$ are related to mass eigenstates by ( $u^{*}$ for antineutrinos)

$$
\nu^{\prime}=u \nu
$$

The transitions between $\nu^{\prime}$ are described by the amplitude matrix

$$
\mathcal{A}=u e^{-i H^{0} t} u^{-1}
$$

In presence of standard matter with arbitrary electron number density, the propagation is usually well aproximated by

$$
i \partial_{t} \nu=\left(H^{0}+\rho(t) u A u^{-1}\right) \nu
$$

where $\mathrm{A}$ is a matrix with $A_{11}$ as only non-zero element. $\rho(t)$, essentially a forward scattering amplitude, is proportional to the electron number density of the medium

$$
\rho(t)= \pm \sqrt{ } 2 G_{F} N_{e}(t)
$$

The plus/minus sign is for neutrinos/antineutrinos respectively.

The difference of the eigenvalues of $H^{0}$ ( the inverse of the usually defined oscillation length) is typically considered to be, in the solar case, of the order

$$
\frac{m_{i}^{2}-m_{j}^{2}}{2 E} \approx \frac{0.1-1 e V^{2}}{1 M e V} \approx 10^{-6}-10^{-7} \mathrm{eV}
$$

The electron number density can be parametrized (data taken from [4]), for sufficiently far distances from the solar core as

$$
N_{e}(r)=N_{0} \exp (-\lambda r)=160 \pm 30 \exp \left(-10.6 \pm 0.2 \mathrm{r} / \mathrm{r}_{0}\right) \mathrm{mol} / \mathrm{cm}^{3}
$$

with $r, r_{0}$ the distance from the center and solar radius respectively. At the solar center

$$
N_{e}(0) \approx 100 \mathrm{~mol} / \mathrm{cm}^{3}
$$


So the value for $\rho(t)$ varies between zero and its maximum value of $\approx 10^{-11} \mathrm{eV}$ at the center.

Later we will see that the following dimensionless quantities play an important role:

$$
\frac{m_{i}^{2}-m_{j}^{2}}{2 E \lambda} \approx 1.7510^{8} \frac{\Delta m^{2}\left(e V^{2}\right)}{E(M e v)}\left(\approx 1 \text { for } \mathrm{E}=1 \mathrm{MeV} \text { and } \Delta m^{2}=10^{-7} e V^{2}\right)
$$

$\rho(t) / \lambda$ varies between $5.510^{3}$ and $\approx 0$ when the neutrino goes outwards from the center to the sun surface.

In the general case Eq.(2) must be solved numerically ([2, 3]). Comparation with numerical studies and qualitative analyses show that for slow (in the neutrino time scale ) and monotonically varying matter density, the adiabatic approximation can be applied except, possibly, in some small "resonance" region (MSW effect). This resonance effect is essentially the same as the non-adiabatic crossing of energy levels in diatomic molecules studied already 60 years ago. The overall transition probability depends essentially of the existence of this resonance region and not on the detailed matter density function. The aproximate transition probability was computed for two species in two classical papers by Landau and Zener [5] supposing that the density varies linearly in the (small) resonance region, its formula, adapted to the neutrino context, is:

$$
P_{e e}^{L Z}=\exp \left[-\pi \frac{\Delta m^{2}}{2 E \lambda} \frac{s^{2} 2 \theta}{c 2 \theta}\right]
$$

The point $t_{r e s}$ where the resonance appears is given by

$$
\rho\left(t_{r e s}\right)=\frac{\Delta m^{2} \cos 2 \theta}{E}
$$

$\theta$ is the 2-neutrino mixing angle.

Apart from this approximate results, exact solutions have appeared for particular forms of the function $\rho$ : for linear densities, in terms of Weber-Hermite functions ([6, []); for functions of the form

$$
\rho(t)=C(1+\tanh (\lambda t))
$$

in [8], and for exponentially decaying densities

$$
\rho(t)=c e^{-\lambda t}
$$

in [9, 10]. All these functions for the density are of interest in the solar case, the last two reproduce rather well the real solar density except in the inner core and to a less degree in the surface boundary. All of these solutions are valid for 2 species and all of them are obtained reducing Eq.(2) to a second order differential equation identifiable with one of the classical equations of the mathematical physics.

In this paper, using a completely different method, summing a standard time-dependent perturbative expansion, we will give an exact, analytical solution for an arbitrary number of species and for arbitrary (possibly non-unitary in case of sterile neutrinos) mixing 
matrix using the exponentially decaying function. Using the limit $t \rightarrow \infty$, which is appropriated for the case of detection at Earth, we will give specially simple solutions (this is something that papers [9, 10] fail to give in the 2-dimensional case).

\section{An exact solution for $\delta(t) \approx \exp (-t)$.}

We will get an exact solution for the neutrino evolution equations in the special case where the electron density decays exponentially. Standard non-stationary perturbation theory will be used; we will give general expressions for the n-th term and sum the full series (regardless of mathematical convergence problems, we will see a posteriori that the series effectively converges, and the result is physically meaningful).

The evolution operator of the differential system

$$
i \partial_{t} \nu=H(t) \nu
$$

is such that

$$
\nu(t)=U\left(t, t_{0}\right) \nu\left(t_{0}\right)
$$

obeying the integral equation

$$
U\left(t, t_{0}\right)=1-i \int_{t 0}^{t} H(\tau) U\left(\tau, t_{0}\right) d \tau
$$

We are interested in the case where the hamiltonian, of finite dimension $\mathrm{k}$, can be decomposed in the following way

$$
H=H^{0}+V(t)=H^{0}+\rho(t) V
$$

$H^{0}$,the free hamiltonian, hermitic and independent of time, has eigenvalues $\left\{E_{1}, \ldots, E_{k}\right\}$ and eigenvectors $\{|a>| b>,\ldots\}$. We can suppose that at least two of the eigenvalues are distinct, the completely degenerate case can be solved in a trivial way. $\rho(t)$ a scalar function and $\mathrm{V}$ a hermitic matrix with all eigenvalues but one equal to zero.

Formally, it is possible to solve Eq.(阿) by succesive iterations:

$$
U\left(t, t_{0}\right)=U^{(0)}\left(t, t_{0}\right)+\sum_{n=1}^{\infty} U^{(n)}\left(t, t_{0}\right)
$$

where the order 0 can be taken as:

$$
U^{(0)}\left(t, t_{0}\right)=\exp \left(-i H^{0}\left(t-t_{0}\right)\right)
$$

and $U^{(n)}$ is the well-known integral

$$
U^{(n)}=(-i)^{n} \int_{\Gamma} d \tau_{n} \ldots d \tau_{1} U^{0}\left(t, \tau_{n}\right) V\left(\tau_{n}\right) \ldots U^{0}\left(\tau_{2}, \tau_{1}\right) V\left(\tau_{1}\right) U^{0}\left(\tau_{1}, t_{0}\right)
$$


The domain of integration is defined by

$$
\Gamma \equiv t>\tau_{n}>\ldots>\tau_{1}>t_{0} .
$$

A $\mathrm{V}$ with the special form of interest to us can be always reduced to the form

$$
V=u^{-1} \operatorname{Diag}(1,0, \ldots, 0) u
$$

for some unitary matrix $\mathrm{u}$ and its elements are given by

$$
V_{i j}=\sum_{k, l} u_{i k}^{-1} \delta_{k 1} \delta_{l 1} u_{l j}=u_{1 j} u_{i 1}^{-1}
$$

The following property will be used later valid for any $\mathrm{V}$ of this form:

$$
V_{i j} V_{j l}=V_{i l} V_{j j} \quad \text { (no summation involved) }
$$

First we will compute $\mathrm{U}$ in the $t \rightarrow \infty$ limit. And the end we will see that in fact we can compute it for any finite $t$ using the properties of the evolution operator. For simplicity, we will work in the basis of eigenstates of $H^{0}$. For practical applications (weak eigenstates neutrino transition probabilities) we may want to apply a straightforward coordinate change $U^{\prime}=v^{-1} U v$ later.

Trought elementary manipulations we get, from Eq.(10),

$$
\begin{aligned}
& <b\left|U^{(n)}\right| a>=(-i)^{n} \exp \left(-i\left(E_{b} t-E_{a} t_{0}\right)\right) \times \\
& \quad \times \sum_{k 1, \ldots, k(n-1)} \int_{\Gamma} d^{n} \tau \exp \left(i \tau_{n} w_{b k 1}+\ldots+i \tau_{1} w_{k(n-1) a}\right) V_{b k 1}\left(\tau_{n}\right) \ldots V_{k(n-1) a}\left(\tau_{1}\right)
\end{aligned}
$$

With $w_{k 1 k 2}=E_{k 1}-E_{k 2}$. Besides, using the special form of the potential:

$$
\begin{gathered}
<b\left|U^{(n)}\right| a>=(-i)^{n} \exp \left(-i\left(E_{b} t-E_{a} t_{0}\right)\right) V_{b a} \sum_{k 1, \ldots, k(n-1)} B_{k 1} \ldots B_{k(n-1)} A_{k 1, \ldots, k(n-1)} \\
A_{k 1, \ldots, k(n-1)}=\int_{\Gamma} d^{n} \tau \exp \left(i \tau_{n} w_{b k 1}+\ldots+i \tau_{1} w_{k(n-1) a}\right) \rho\left(\tau_{n}\right) \ldots \rho\left(\tau_{1}\right) \\
B_{k}=V_{k k}
\end{gathered}
$$

The multiple sums run over all the eigenvalues of the hamiltonian independently.

We consider an idealized exponentially decaying electron density profile for the sun:

$$
\rho(t)=\rho_{0} \exp (-\lambda t)
$$

Where $\rho_{0}$ can be positive or negative. Redefining $E_{k}, \rho_{0}$ we can always suppose $\lambda=1$. 
For this kind of density we can use the following equality,

$$
\begin{aligned}
I_{n}\left(w_{1}, \ldots, w_{n}\right) \equiv & \int_{t 0}^{\infty} \ldots \int_{t 0}^{x_{n-1}} d x_{1} \ldots d x_{n} \exp \sum_{n} w_{n} x_{n} \\
= & \frac{(-1)^{n} \exp \left(t_{0} \sum_{n} w_{n}\right)}{w_{n}\left(w_{n}+w_{n-1}\right) \ldots\left(w_{n}+w_{n-1} \ldots+w_{1}\right)} \\
& \left(\text { valid if } \Re w_{n}<0, \forall n\right)
\end{aligned}
$$

This equality can be proved by induction, noting that, with the help of the variable change

$$
x_{1}=y_{1}+y_{2} ; \quad x_{2}=y_{2}
$$

the following recurrence relation holds

$$
I_{n}\left(w_{1}, \ldots, w_{n}\right)=I_{1}\left(w_{1}\right) \times I_{n-1}\left(w_{1}+w_{2}, w_{3}, \ldots, w_{n}\right)
$$

that together with

$$
I_{1}(w)=\frac{-\exp \left(w t_{0}\right)}{w}
$$

proves our result.

In our case

$$
\begin{aligned}
w_{n}+w_{n-1}+\ldots+w_{n-j+1} & =i w_{b k 1}-1+i w_{k 1 k 2}-1+\ldots+i w_{k(j-1) k j}-1 \\
& =i w_{b k j}-j
\end{aligned}
$$

so the (n-1)-tensor $A_{i . . j}$ can be factorized

$$
A_{k 1, k 2, \ldots, k(n-1)}=e^{i w_{b a} t_{0}} \frac{\left(-\rho_{0} e^{-t_{0}}\right)^{n}}{i w_{b a}-n} A_{k 1} A_{k 2} \ldots A_{k(n-1)}
$$

with

$$
A_{k(m)}=\frac{1}{i w_{b k(m)}-m}
$$

the fact that this factorization is possible is the key for the solution of the problem. Note also the importance of the ordering in the definition of the A's.

So,

$$
\sum B_{k 1} \ldots B_{k(n-1)} A_{k 1} \ldots A_{k(n-1)} \propto \prod_{m=1}^{n-1} \sum_{\text {all eigenvalues }} B_{k(m)} A_{k(m)} \equiv \prod_{m} f_{m}
$$

What remains is the computation of the expression $\prod_{m} f_{m}$. Such computation is easy even in a general case but rather messy. We will do it first for the 2-dimensional case for the sake of clarity. 


\subsubsection{2-dimensional case}

For a two-dimensional case and taking $b=1$

$$
f_{m}=\frac{B_{1}}{-m}+\frac{B_{2}}{i w_{12}-m}=\frac{-1}{m} \frac{\left(-i B_{1} w_{12}+m\right)}{\left(-i w_{12}+m\right)}
$$

and the product of them

$$
\prod_{m=1}^{n-1} f_{m}=\frac{1}{B_{1}} \frac{(-1)^{n-1}}{(n-1) !} \frac{\left(B_{1} \beta\right)_{(n)}}{(\beta)_{(n)}}
$$

where we have used the Pochammer symbol Eq. (69) and defined $\beta=-i w_{12}$. Inserting this product in the previous expressions and recalling the series expansion of the Confluent Hypergeometric function Eq. (68) we can write immediately the value of the diagonal elements of $U$

$$
\begin{aligned}
& <b|U| b>= \\
& =e^{-i E_{b}\left(t-t_{0}\right)}\left(1+V_{b b} \sum_{n=1}(-i)^{n} \frac{\left(-\rho_{0} \exp \left(-t_{0}\right)\right)^{n}}{-n} \prod_{m=1}^{n-1} f_{m}\right) \\
& =e^{-i E_{b}\left(t-t_{0}\right)}\left(1+\frac{V_{b b}}{B_{b}} \sum_{n=1} \frac{\left(-i \rho_{0} \exp \left(-t_{0}\right)\right)^{n}}{n !} \frac{\left(B_{b} \beta\right)_{(n)}}{\beta_{(n)}}\right) \\
& =e^{-i E_{b}\left(t-t_{0}\right)}{ }_{1} F_{1}\left(B_{b} \beta, \beta, z\right)
\end{aligned}
$$

here $\beta=-i w_{b a}$ and $z=-i \rho_{0} \exp \left(-t_{0}\right)$.

For the general case $a \neq b$ is neccesary to work a little bit more to give a closed expression. In this case it appears an additional factor in each term of the series which doesn't allow for the immediate identification with any known function:

$$
\begin{aligned}
& <b|U| a>= \\
& =e^{-i\left(E_{b} t-E_{a} t_{0}\right)}\left(\delta_{a b}+e^{i\left(E_{b}-E_{a}\right) t_{0}} V_{b a} \sum_{n=1}(-i)^{n} \frac{\left(-\rho_{0} \exp \left(-t_{0}\right)\right)^{n}}{-\beta-n} \prod_{m=1}^{n-1} f_{m}\right) \\
& =e^{-i\left(E_{b} t-E_{a} t_{0}\right)} e^{i\left(E_{b}-E_{a}\right) t_{0}} \frac{V_{b a}}{B_{b}} \sum_{n} \frac{z^{n}}{(n-1) !} \frac{1}{\beta+n} \frac{\left(B_{b} \beta\right)_{(n)}}{\beta_{(n)}} \\
& =e^{-i E_{b}\left(t-t_{0}\right)} \frac{V_{b a}}{B_{b}} g\left(B_{b} \beta, \beta ; z\right)
\end{aligned}
$$

as before here $\beta=-i w_{b a}$.

The function

$$
g(z)=\sum_{n=1}^{\infty} \frac{1}{\beta+n} \frac{(\alpha \beta)_{(n)}}{(\beta)_{(n)}} \frac{z^{n}}{(n-1) !}
$$


is well defined, the series is absolutely convergent for all $\mathrm{z}$; manipulating termwise, and using the Eq.(73) we get the following differential equation for it

$$
z g^{\prime}+\beta g=\alpha z_{1} F_{1}(1+\alpha \beta, 1+\beta ; z) ; \quad g(0)=0
$$

whose solution is

$$
g(z)=\alpha z^{-\beta} \int_{0}^{z} y^{\beta}{ }_{1} F_{1}(1+\alpha \beta, 1+\beta ; y) d y
$$

Using the Expression (71) for the special case $c=\gamma+1$, we finally write (note that in our case the conditions of validity of the previous integral are fullfilled) :

$$
\begin{aligned}
g(z) & =\frac{\alpha}{1+\beta} z{ }_{1} F_{1}(1+\alpha \beta, 2+\beta ; z) \\
& ={ }_{1} F_{1}(\alpha \beta, \beta ; z)-{ }_{1} F_{1}(\alpha \beta, 1+\beta ; z)
\end{aligned}
$$

The remarkable second equality can be deduced from the expressions (73-77). So

$$
\begin{aligned}
< & b|U| a>= \\
& =e^{-i E_{b}\left(t-t_{0}\right)} \frac{V_{b a}}{B_{b}} \frac{B_{b}}{1+\beta} z{ }_{1} F_{1}\left(1+B_{b} \beta, 2+\beta ; z\right) \\
< & a|U| b>= \\
& =e^{-i E_{a}\left(t-t_{0}\right)} \frac{V_{a b}}{B_{a}} \frac{B_{a}}{1-\beta} z{ }_{1} F_{1}\left(1-B_{a} \beta, 2-\beta ; z\right) \\
& =e^{-i E_{a}\left(t-t_{0}\right)} \frac{V_{a b}}{1-\beta} z e^{z}{ }_{1} F_{1}\left(1-\left(1-B_{a}\right) \beta, 2-\beta ;-z\right) \\
& =e^{-i E_{a}\left(t-t_{0}\right)} \frac{V_{a b}}{1-\beta} z e^{z}{ }_{1} F_{1}\left(1-B_{b} \beta, 2-\beta ;-z\right) \\
& =e^{-i E_{a}\left(t-t_{0}\right)} \frac{V_{a b}}{1-\beta} z e^{z}{ }_{1} F_{1}{ }^{*}\left(1+B_{b} \beta, 2+\beta ;-z^{*}\right) \\
& =e^{-i E_{a}\left(t-t_{0}\right)} \frac{V_{a b}}{1-\beta} z e^{z}{ }_{1} F_{1}{ }^{*}\left(1+B_{b} \beta, 2+\beta ; z\right)
\end{aligned}
$$

Where we have used the Eq.(73). The last Formula (29) is only valid for $z$ purely imaginary, in the case of general complex $z$ (for example given $\rho_{0}$ complex ) Formula (28) is valid.

We have completed so the computation of U. In summary, the elements of the operator $\mathrm{U}$ in a basis of eigenvectors of $H^{0}$ are

$$
\begin{aligned}
U\left(t, t_{0}\right) & =\exp -i H_{0}\left(t-t_{0}\right) U_{\text {red }}\left(\rho_{0}, t_{0}\right) \\
U_{\text {red }}\left(\rho_{0}, t_{0}\right) & =\left(\begin{array}{cc}
F & \frac{V_{12}}{V_{11}} G \\
-\frac{V_{21}}{V_{11}} \exp (z) G^{*} & \exp z F^{*}
\end{array}\right)
\end{aligned}
$$


with the shorthands

$$
G=g(z)=\frac{V_{11}}{1+\beta}{ }_{1} F_{1}\left(1+B_{1} \beta, 2+\beta ; z\right), \quad F={ }_{1} F_{1}\left(B_{1} \beta, \beta ; z\right)
$$

As it should be, $U(t)$ obeys the free equation:

$$
i \partial_{t} U(t)=H^{0} U(t)
$$

For a reverse sign in $\rho_{0}$ (antineutrino case), the matrix $U_{\text {red }}$ is basically the same except for the substitution $B_{1} \rightarrow 1-B_{1}$ :

$$
U_{\text {red }}\left(-\rho_{0}, t_{0}\right)=\exp (-z)\left(\begin{array}{cc}
F^{*} & -\frac{V_{12}}{V_{11}} G^{*} \\
\frac{V_{21}}{V_{11}} \exp (z) G & \exp z F
\end{array}\right)
$$

where now $\mathrm{F}$ stands for ${ }_{1} F_{1}\left(\left(1-B_{1}\right) \beta, \beta, z\right)$, and $\mathrm{G}$ changes similarly.

For $\mathrm{H}$ hermitic $\mathrm{U}$ is unitary and we get as a byproduct the following non-trivial identity for the absolute value of Hypergeometric functions:

$$
\left\|_{1} F_{1}(\alpha \beta, \beta ; z)\right\|^{2}+\alpha(1-\alpha)\left\|\frac{z}{1+\beta}{ }_{1} F_{1}(1+\alpha \beta, 2+\beta ; z)\right\|^{2} \equiv 1 \quad(\alpha \text { real })
$$

or, using the second Equality in (27)

$$
\left|{ }_{1} F_{1}(\alpha \beta, \beta ; z)\right|^{2}+\left(\frac{1-\alpha}{\alpha}\right)\left|{ }_{1} F_{1}(\alpha \beta, 1+\beta ; z)-{ }_{1} F_{1}(\alpha \beta, \beta ; z)\right|^{2} \equiv 1 \quad(\alpha \text { real })
$$

For the particular case of $\mathrm{u}$ being the orthogonal 2-dimensional matrix

$$
u=\left(\begin{array}{cc}
C \theta & -S \theta \\
S \theta & C \theta
\end{array}\right)
$$

the matrix $\mathrm{V}$ is

$$
V=\left(\begin{array}{cc}
C^{2} \theta & -S \theta C \theta \\
-S \theta C \theta & S^{2} \theta
\end{array}\right)
$$

and $H^{0}$ the standard neutrino oscillation hamiltonian given by Eq.(1). We arrive to $\left(t_{0}=0\right.$ and restoring $\lambda$ ),

$$
\begin{aligned}
U_{11}^{r e d} & ={ }_{1} F_{1}\left(\frac{i w_{21}}{\lambda} C^{2} \theta, \frac{i w_{21}}{\lambda} ; \frac{-i \rho_{0}}{\lambda}\right) \\
U_{12}^{r e d} & =T \theta \frac{-i \rho_{0} / \lambda}{1+i w_{21} / \lambda}{ }_{1} F_{1}\left(1+\frac{i w_{21}}{\lambda} C^{2} \theta, 2+\frac{i w_{21}}{\lambda} ; \frac{-i \rho_{0}}{\lambda}\right)
\end{aligned}
$$

We are interested in the expression of the evolution operator in the basis of eigenvalues of $\mathrm{V}$, it is straigthforward to compute $U^{\prime}=u U u^{-1}$. After some algebra, applying the 
identities we have seen before and averaging out time dependent terms, the probability of electron permanence is

$$
P_{e e}=\left\|U_{11}^{\prime}\right\|^{2}=1-S^{2} \theta\left(1+C 2 \theta\left\|{ }_{1} F_{1}\left(\frac{i w_{21}}{\lambda} C^{2} \theta, 1+\frac{i w_{21}}{\lambda} ; \frac{-i \rho_{0}}{\lambda}\right)\right\|^{2}\right)
$$

An expression equivalent to the previous one has been obtained already by [11] in terms of Whittaker functions. However our expression is simpler and more compact because we use the infinite time limit.

In the limit $\rho_{0} \rightarrow 0$ or free case, $z \rightarrow 0$ and the hypergeometric functions go to $1, \mathrm{U}$ becomes as expected:

$$
U=\exp -i H^{0} t
$$

In the limit of vanishing mixing angle, $C^{2} \theta \rightarrow 1, \tan \theta \rightarrow 0$ and ${ }_{1} F_{1}(z) \rightarrow \exp z$ in (34), so

$$
P_{e e}=1
$$

In the limit $w_{21} / \lambda=\Delta m^{2} / 2 E \lambda \rightarrow 0,{ }_{1} F_{1} \rightarrow 1$ in (36) and

$$
P_{e e}=1-\frac{1}{2} S^{2} 2 \theta
$$

In the the limit $w_{21} / \lambda>>1$ (adiabatic regime) we distinguish two cases: if $w_{21} / \lambda \not|z|$ we have ${ }_{1} F_{1} \rightarrow \exp \left(-i C^{2} \theta \rho_{0} / \lambda\right)$ and Formula (38) also applies; if $w_{21} / \lambda \simeq|z|$ a resonance occurs and the probability drops abruptly. Some asymptotic formulas exist for this case valid except in the central region of the resonance (see [12, 13]).

In the limit of small (but not vanishing) mixing angle, we are left in principle with the expression for the electron permanence probability (computed ignoring diagonal terms in $\mathrm{U})$

$$
P_{e e}=\left(1-2 C^{2} \theta S^{2} \theta\right)\left\|_{1} F_{1}\left(\frac{i w_{21}}{\lambda} C^{2} \theta, \frac{i w_{21}}{\lambda} ; \frac{-i \rho_{0}}{\lambda}\right)\right\|^{2}
$$

In the figures we show a comparison between formulas (36) and (39). We see that even at very small angles Eq.(39) is not a very good approximation of (36) as we could expect.

In Figure (11) we plot $\left|{ }_{1} F_{1}\right|^{2}$ (Eq.(39)) as a function of its parameters keeping $\mathrm{z}$ constant. Increasing values of the x-axis implies a larger mass difference or a smaller neutrino energy. We observe that the probability is near 1 ( adiabatic evolution ) except for a very concrete region of the parameter space (MSW effect or existence of a resonance layer). The starting point and width of this region (but not its end point) depends on the parameters involved, in particular $\cos ^{2} \theta$.

Figure (2) is equivalent to Fig.(11), this time we plot the exact expression for the e-e probability, Eq.(36), The global behaviour is exactly the same as before, the local oscillations have disappeared now however. Figure (3) show the behaviour of the resonance region also for large mixing angles. The continuos curve is our exact solution, the dashed 
curve the Parker approximate formula as given in ([14]). Figure (伍) show the behaviour for antineutrinos (reverse sign for $\rho_{0}$ in $\mathrm{z}$ ), in this case the resonance is absent.

In a second set of plots (figs.(5,6)) we keep constant the parameters and vary $\mathrm{z}$ or equivalently the production point of the neutrino: larger $|z|$ or $\rho_{0}$ means larger density at the creation point which implies that this one is nearer to the center. We see again there is a transition between two well defined zones: for a very far creation point, the neutrino doesn't pass through any resonance layer, adiabatic regime conditions always apply and $P_{e e} \sim 1$.

In order to study in detail the existence and properties of the resonance region it would be convenient to have general formulas for the zeros of the Hypergeometric functions, as function not only of its argument but also of its parameters. Unfortunely very little is known in a general case. There are some results (see appendix and reference therein) about the real zeros of ${ }_{1} F_{1}(a, b ; x)$ with $a, b$ real.

We note that the expressions (30) and (39) are still formally valid for a purely imaginary $\lambda$ (we take for granted that we can take convenient limits $\Re w_{n} \rightarrow 0$ in the integral (15)). Expression (36) however is not valid any more because we used the hermiticity of $\mathrm{H}$ (and unitarity of U) in computing it. The extrema of $\left|{ }_{1} F_{1}\right|^{2}$ are given essentially by the zeros of ${ }_{1} F_{1}\left(1+a, 1+b ; x_{j}\right)$; for $\lambda$ purely imaginary, argument and parameters in ${ }_{1} F_{1}$ become real and we can apply the bounds in Appendix A given by the Eqs. (78-80) . This bounds can be used to deduce the regions in the parameter space which allow for the existence of such extrema.

\subsubsection{3- and k-dimensional cases}

For three neutrino species, we can write similarly to Eq.(20)

$$
f_{m}=\frac{B_{1}}{-m}+\frac{B_{2}}{i w_{12}-m}+\frac{B_{3}}{i w_{13}-m}=\frac{-1}{m} \frac{\left(-a_{1}+m\right)\left(-a_{2}+m\right)}{\left(-i w_{12}+m\right)\left(-i w_{13}+m\right)}
$$

where $a_{1}, a_{2}$ are the roots of a certain 2-degree polynomial, they obey:

$$
\left\{\begin{aligned}
a_{1}+a_{2} & =i\left(w_{12}\left(1-B_{2}\right)+w_{13}\left(1-B_{3}\right)\right) \\
a_{1} a_{2} & =-B_{1} w_{12} w_{13}
\end{aligned}\right.
$$

and the product of them

$$
\prod_{m=1}^{n-1} f_{m}=\frac{1}{B_{1}} \frac{(-1)^{n-1}}{(n-1) !} \frac{\left(-a_{1}\right)_{(n)}\left(-a_{2}\right)_{(n)}}{\left(\beta_{1}\right)_{(n)}\left(\beta_{2}\right)_{(n)}}
$$

with $\beta_{1,2}=-i w_{12,13}$. In the simplification of the previous equation has been important the expression for $a_{1} a_{2}$ in Eq.(41). Note that this last expression is only valid if $w_{12}$ and $w_{13}$ are both different from zero.

For the diagonal elements of $\mathrm{U}$, we can write immediately, in terms of the generalized Hypergeometric functions (Definition (70)) 


$$
<b|U| b>=e^{-i E_{b}\left(t-t_{0}\right)}{ }_{2} F_{2}\left(-a_{1},-a_{2},-i w_{b, k 1},-i w_{b, k 2} ; z\right)
$$

with

$$
\left\{\begin{aligned}
a_{1}+a_{2} & =i\left(w_{b, k 1}\left(1-B_{k 1}\right)+w_{b, k 2}\left(1-B_{k 2}\right)\right) \\
a_{1} a_{2} & =-B_{b} w_{b, k 1} w_{b, k 2}
\end{aligned}\right.
$$

$k_{1}, k_{2}$ are labels for the two eigenvectors different from $b$.

For the $<b|U| a>(a \neq b)$, we can repeat exactly the same path as we did in the 2-dimensional section. We arrive to (we do it already for the general case):

$z g^{\prime}+\beta_{1} g=\frac{a_{1} \ldots a_{k-1}}{\beta_{1} \ldots \beta_{k-1}} z_{2} F_{2}\left(-a_{1}+1, \ldots,-a_{k-1}+1 ; \beta_{1}+1, \ldots, \beta_{k-1}+1 ; z\right), \quad g(0)=0$

with $\beta_{1}=-i w_{b a}, \beta_{k}=-i w_{b k}$, where $k \neq a, b$. The solution of which is

$$
\begin{aligned}
g(z) & =\frac{a_{1} \ldots a_{k-1}}{\beta_{1} \ldots \beta_{k-1}} z^{-\beta_{1}} \int_{0}^{z} d w w_{k-1}^{\beta_{1}} F_{k-1}\left(-a_{1}+1, \ldots,-a_{k-1}+1 ; \beta_{1}, \ldots, \beta_{k-1} ; w\right) \\
& =\frac{a_{1} \ldots a_{k-1}}{\beta_{1} \ldots \beta_{k-1}} z \int_{0}^{1} d s s_{k-1}^{\beta_{1}} F_{k-1}\left(-a_{1}+1, \ldots,-a_{k-1}+1 ; \beta_{1}, \ldots, \beta_{k-1} ; s z\right) \\
& =\frac{a_{1} \ldots a_{k-1}}{\beta_{1} \ldots \beta_{k-1}} \frac{z}{1+\beta_{1}}{ }_{k-1} F_{k-1}\left(-a_{1}+1, \ldots,-a_{k-1}+1 ; \beta_{1}+2, \beta_{2}+1, \ldots, \beta_{k-1}+1 ; z\right)
\end{aligned}
$$

Where we have used the expression Eq.(72). for $\mu=1$

So

$$
<b|U| a>=e^{-i\left(E_{b} t-E_{a} t_{0}\right)} V_{b a} \frac{z}{1+i w_{b a}}{ }_{2} F_{2}\left(-a_{1}+1,-a_{2}+1 ;-i w_{b, k 1}+2,-i w_{b, k 2}+1 ; z\right)
$$

$a_{1}, a_{2}$ fullfill the equations (44) with $k_{1}=a, k_{2}$ are labels for the eigenvectors different to $b$.

Let's take for u a general unitary 3-dimensional parametrized as follows

$$
u=\left(\begin{array}{ccc}
1 & 0 & 0 \\
0 & C \psi & S \psi \\
0 & -S \psi & C \psi
\end{array}\right)\left(\begin{array}{ccc}
1 & 0 & 0 \\
0 & e^{i \delta} & 0 \\
0 & 0 & e^{-i \delta}
\end{array}\right)\left(\begin{array}{ccc}
C \phi & 0 & S \phi \\
0 & 1 & 0 \\
-S \phi & 0 & C \phi
\end{array}\right)\left(\begin{array}{ccc}
C \omega & S \omega & 0 \\
-S \omega & C \omega & 0 \\
0 & 0 & 1
\end{array}\right)
$$

The potential $\mathrm{V}$ is

$$
V_{a b} \equiv u_{1 b} u_{a 1}^{-1}=u_{1 b} u_{1 a}
$$

The last equality is valid only in our particurlar case (or for any real u). 


$$
u_{1 a}(a=1-3)=(C \phi C \omega, C \phi S \omega, S \phi)
$$

Note that the phase $\delta$ and the angle $\psi$ don't appear in V.

In the basis where $\mathrm{U}$ is diagonal $U^{\prime}=u U u^{-1}$. In particular, for the e-e transition we have

$$
\mathcal{A}_{11}=U_{11}^{\prime}=\sum_{k l} u_{1 l}\left(u^{-1}\right)_{k 1} U_{l k}=\sum_{k l} V_{k l} U_{k l}=\operatorname{tr} U V
$$

In the small mixing limit the terms $V_{k l}$ are nearly zero for $k, l \neq 1$. Averaging out time dependent terms, we write approximately:

$$
P_{e e}=\left|U_{11}^{\prime}\right|^{2} \simeq C^{2} \phi C^{2} \omega\left|U_{11}\left(={ }_{2} F_{2}\right)\right|^{2}
$$

with obvious arguments for the hypergeometric function. The computation of a exact, fully simplified expression for $U_{11}^{\prime}$ requires the algebraic manipulation of several ${ }_{2} F_{2}$ functions and is not of major interest. For the expected degree of approximation of this formula, see the comments correspondent to the 2-dimensional case.

Now we are going to study the behaviour of $U_{11}$ for two different limits. Let's suppose that the first and second eigenvalues are nearly degenerate: $w_{12} \rightarrow 0$, in this case

$$
\left\{\begin{array}{l}
a_{1}=i w_{13}\left(1-B_{3}\right) \\
a_{2}=-B_{1} w_{12} w_{13} \rightarrow 0
\end{array}\right.
$$

and

$$
\begin{aligned}
U_{11} & \propto 1-\frac{a_{2}}{w_{12}}\left({ }_{1} F_{1}\left(-a_{1},-i w_{13} ; z\right)-1\right) \\
& =1-B_{1} w_{13}\left(1-{ }_{1} F_{1}\left(-i w_{13}\left(1-B_{3}\right),-i w_{13} ; z\right)\right.
\end{aligned}
$$

The 3-oscillations has been reduced essentially to a 2 dimensional problem. We can apply to this last formula the arguments of previous section for finding different limiting behaviours as function of the size of $w_{13}$.

It is interesting also to study what happens when one of the eigenvalues is much bigger than the others (but none goes to zero), so some of the w's goes to infinity. Let's suppose $w_{13} \rightarrow \infty$. In such a limit, solving previously the second degree algebraic equation, we have

$$
\left\{\begin{array}{l}
a_{1}=\frac{i w_{12}\left(1-B_{2}\right)}{2} \\
a_{2}=i w_{13}\left(1-B_{3}\right)=i w_{13}\left(B_{1}+B_{2}\right)
\end{array}\right.
$$

Thus

$$
\begin{aligned}
\lim _{w_{13} \rightarrow \infty} U_{11} & \propto \lim _{w_{13} \rightarrow \infty}{ }_{2} F_{2}\left(\frac{i w_{12}\left(1-B_{2}\right)}{2}, i w_{13}\left(B_{1}+B_{2}\right), i w_{12}, i w_{13} ; z\right) \\
& ={ }_{1} F_{1}\left(\frac{i w_{12}\left(1-B_{2}\right)}{2}, i w_{12} ; z\left(B_{1}+B_{2}\right)\right)
\end{aligned}
$$


so, as expected, the system behaves effectively as a 2-dimensional system. Note that the 2-dimensional submatrix of $\mathrm{u}$ is not unitary, so the sum $B_{1}+B_{2}$ is not equal to unity anymore in Eq.(53). This procedure is specially useful for the study of oscillations in models with extremely heavy sterile neutrinos as those inspired in SO(10) GUT theories.

For k dimensions, we can write similarly to Eq. (20)

$$
f_{m}=\frac{B_{1}}{-m}+\frac{B_{2}}{i w_{12}-m}+\ldots+\frac{B_{k}}{i w_{1 k}-m}=\frac{(-1)^{k}}{m} \frac{\left(\left(-a_{1}\right)+m\right) \ldots\left(\left(-a_{k-1}\right)+m\right)}{\left(-i w_{12}+m\right) \ldots\left(-i w_{1 k}+m\right)}
$$

where $a_{1}, \ldots, a_{k-1}$ are the roots of the (k-1)-degree polynomial,

$$
p(m)=\sum_{s=1}^{k} B_{s} \prod_{j \neq s}\left(m-i w_{1, j}\right)
$$

The coefficient of the power of greatest degree, $c_{0}$, and the constant term, $c_{k-1}$ of this polynomial are:

$$
\begin{gathered}
c_{0}=\sum_{s} B_{s}=1 \\
c_{k-1}=p(0)=\sum_{s=1}^{k} B_{s} \prod_{j \neq s}(-i) w_{1, j}=(-i)^{k-1} B_{1} \prod_{j \neq 1} w_{1 j}
\end{gathered}
$$

the product of the roots is then

$$
a_{1} \cdot \ldots \cdot a_{k-1}=(-1)^{k-1} \frac{c_{k-1}}{c_{1}}=i^{k-1} B_{1} \prod_{j \neq 1} w_{1 j}
$$

Based in the previous formula and assuming all the $w_{i j}$ different from zero, we can generalize the 2 and 3-dimensional expressions for the product,

$$
\prod_{m=1}^{n-1} f_{m}=\frac{1}{B_{1}} \frac{(-1)^{n}}{(n-1) !} \frac{\left(-a_{1}\right)_{(n)} \ldots\left(-a_{k-1}\right)_{(n)}}{\left(\beta_{1}\right)_{(n)} \ldots\left(\beta_{k-1}\right)_{(n)}}
$$

with $\beta_{s}=-i w_{b s}$.

As it can be deduced trivially, the matrix $\mathrm{U}$ for $\mathrm{k}$ dimensions is the product

$$
U(t)=\exp -i H_{0}\left(t-t_{0}\right) U_{r e d}\left(H^{0}, V, \rho_{0}, t_{0}\right)
$$

The elements of $U_{\text {red }}$ being essentially Generalized Confluent Hypergeometric Functions of order k-1. The diagonal elements are given in a basis of eigenvalues of $H^{0}$ by:

$$
<a\left|U_{\text {red }}\right| a>={ }_{k-1} F_{k-1}\left(-a_{1}, \ldots,-a_{k-1}, \beta_{1}, \ldots, \beta_{k-1} ; z\right)
$$

with the $\alpha^{\prime} s$ and $\beta$ 's defined as before, and $z=-i e^{-t 0 / \lambda} \rho_{0} / \lambda$.

For non-diagonal elements: 


$$
<b\left|U_{r e d}\right| a>=\frac{V_{b a}}{V_{b b}} \frac{z}{1+\beta_{1}}{ }_{k-1} F_{k-1}\left(-a_{1}+1, \ldots,-a_{k-1}+1 ; \beta_{1}+2, \ldots, \beta_{k-1}+1 ; z\right)
$$

Note that for each individual element $\beta$ 's and $\alpha$ 's are differently defined.

As it was pointed out before the previous expressions are valid only for the completely non-degenerate case: all the $w_{i j}$ different from zero. The general case will be treated elsewhere, it is easy to see that the order of the hypergemetric functions appearing there will decrease in one unit for each of the w's equal to zero.

The unitarity of $\mathrm{U}$ for any hermitian $\mathrm{H}$ induces a tower of identities between the absolute values of the Hypergeometric Functions generalizing the expressions (32, 33) found for the 2-dimensional case.

\subsubsection{Evolution for finite time.}

Until now we have considered the evolution of the system from a finite $t_{0}$ until infinite time. Now, using general properties of the evolution operator, we will compute the evolution for arbitrary finite intervals.

Any $\mathrm{U}$, for any intermediate time $t_{1}$, obeys the composition property:

$$
U\left(t, t_{0}\right)=U\left(t, t_{1}\right) U\left(t_{1}, t_{0}\right)
$$

Taking the limit $t \rightarrow \infty$ in the above expression we arrive (taking $t_{0}=0$ for simplicity)

$$
U_{\infty}(t, 0)=U_{\infty}\left(t, t_{1}\right) U\left(t_{1}, 0\right)
$$

By the subindex $\infty$ we want to remark we have computed $U$ as in the previous sections, using some limiting process. So, as $U$ has an inverse,

$$
\begin{aligned}
U(t, 0) & =U_{\infty}^{-1}(\mu, t) U_{\infty}(\mu, 0) \\
& =U_{\infty}^{\dagger}(\mu, t) U_{\infty}(\mu, 0) \\
& =U_{r e d}^{\dagger}\left(t_{0}=t\right) e^{i H_{0}(\mu-t)} e^{-i H_{0} \mu} U_{r e d}\left(t_{0}=0\right) \\
& =U_{r e d}^{\dagger}\left(t_{0}=t\right) e^{-i H_{0} t} U_{\text {red }}\left(t_{0}=0\right)
\end{aligned}
$$

Where we have effectuated some relabing to let clear the time dependence. $\mu$, appearing at first as a new arbitrary parameter, disappears from the last expression. $U_{\text {red }}$ is the matrix depending on the diverse parameters of the system, including $t_{0}$ (compare with Eq. (30)).

The matrix defined by Eq. (59) satisfies by construction the initial condition $U(0,0)=$ 1. In addition we must check it satisfies the Equation (4): inserting the last expression we obtained, and eliminating the common factor $U_{\text {red }}\left(t_{0}=0\right)$, we get 


$$
\begin{aligned}
-i \partial_{t} e^{i H_{0} t} U_{\text {red }} & =e^{i H_{0} t} U_{\text {red }}\left(H_{0}+\rho(t) V\right) \\
e^{i H_{0} t} H_{0} U_{\text {red }}-i e^{i H_{0} t} \partial_{t} U_{r e d} & =e^{i H_{0} t} U_{\text {red }}\left(H_{0}+\rho(t) V\right) \\
{\left[H_{0}, U_{r e d}\right] } & =i \partial_{t} U_{\text {red }}+\rho(t) U_{\text {red }} V
\end{aligned}
$$

the dependence of $U_{\text {red }}$ on t comes from the substitution $t_{0}=t$.

If

$$
\left[H_{0}, U_{\text {red }}(t)\right] \approx 0
$$

for example if the difference of the eigenvalues of $H_{0}$ is relatively small, then Eq. (60) has the trivial solution:

$$
U_{\text {red }}(t)=\exp -i V \int_{0}^{t} g(\mu) d \mu
$$

and the U matrix becomes

$$
U(t, 0)=\exp -i H_{0} t U_{r e d}(t)=\exp -i H_{0} t \exp -i V \int_{0}^{t} g(\mu) d \mu
$$

this is a highly non-trivial expression valid for arbitrary $H_{0}, V$ and $g(t)$. Some pertubation approximation expansion in $\Delta E$ can be implemented for better approximations.

In our particular case it is easy to check that the previous Eq. (60) is really fullfilled. It converts to a system of algebraic- functional relations between Hypergeometric functions. For simplicity we take the 2-dimensional case. Eq. (60) is equivalent to only two independent relations, with the same notation as in previous sections:

$$
\begin{aligned}
\partial_{z} F & =F V_{11}+\left|V_{12}\right|^{2} G \\
F & =\left(-V_{12}+\beta / z\right) G+\partial_{z} G
\end{aligned}
$$

with the help of Eq.(73), we obtain instead two algebraic equations that can be proved to be identities with Eqs.(77). We just prove in detail the first one. First we identify $V_{11} \equiv \alpha, V_{12}^{2}=\alpha(1-\alpha)$, so the first term is

$$
\partial_{z} F(\alpha \beta, \beta, z)=\alpha_{1} F_{1}(\alpha \beta+1, \beta+1 ; z)
$$

the second term is

$$
\begin{aligned}
F V_{11}+\left|V_{12}\right|^{2} G & =\alpha_{1} F_{1}(\alpha \beta, \beta ; z)+(1-\alpha)\left({ }_{1} F_{1}(\alpha \beta, \beta ; z)-{ }_{1} F_{1}(\alpha \beta, \beta+1 ; z)\right) \\
& ={ }_{1} F_{1}(\alpha \beta, \beta ; z)-(1-\alpha){ }_{1} F_{1}(\alpha \beta, \beta+1 ; z)
\end{aligned}
$$


In the first line we have used Eq. (33). The last expression is just the identity (76).

In summary, we have proved that the evolution operator for any finite time is

$$
U(t, 0)=U_{r e d}^{\dagger}(t) e^{-i H_{0} t} U_{\text {red }}(0)
$$

with $U_{\text {red }}$ given in the previous sections. Expressions can be given now for the transition probabilities in the mass or weak basis as we did before. However the expressions are rather involved and not so illuminating. For almost any practical purpose we can stick to the expressions given in the $t \rightarrow \infty$ limit.

\section{Conclusions and further discussion.}

Exact expressions for the transition probabilities of neutrinos propagating radially in the sun are given. These are valid for an arbitrary number of neutrino species. The solution, very compact in terms of Hypergeometric Functions, is very suitable for analytical studies of resonance and for systematic numerical approximations. The computational task of computing numerically this kind of functions makes however very impractical the actual use of them in concrete applications for the moment.

The perturbative expansion method used, that in this particular case gives an exact solution, is well suited to produce approximate results for the same matrix form of the potential (all eigenvalues but one equal to zero) but for arbitrary form of $\rho(t)$. The consideration of a finite number of terms in the expansion is expected not to be useful but yes the summation for all orders of appropriate leading terms. Another possibility is to consider the difference between an arbitrary $\rho(t)$ and our exponential as small and do perturbation theory around our exact solution. In principle we expect this procedure to be convenient at far distances from the creation point, as we have seen the probability transition depends more on the existence of a resonant region than on the detailed shape of the potential. This would make possible to compute formulas for non-radial propagation and for the regions where the exponentially decaying density lose validity: central core and solar surface.

The phenomenon of resonance (or MSW effect) is remarkable in itself. The present solution offers a starting point for its analytical description. We note the similarity of this effect with the original form of the Anderson Localization effect presented in [15]. This similarity is specially evident under the treatment done in this work. In both cases the locking of a system in one of its quantum states except for a certain range of the parameter space arise as a property of the solution of a coupled system of ordinary differential equations. As in its case (which physically correspond to the evolution of a particular site in a random lattice) the proper energies (or its differences) of each mode play an important role, however here these are not needed to be stochatically distributed. This condition of randomness is essential in [15], the results in this work induce us to think whether such randomness is a mere mathematical convenience more than having a deep physical meaning. 
Apart from physical interest, our solution has intrinsic mathematical interest in the Theory of Differential equations and of Special Functions. A general theory of equations of the form

$$
\partial_{t} x=(A+f(t) B) x
$$

with A,B constant matrix is missing in spite of the fact these systems are the "next-step" in complexity from all-constant coefficient equations. Only solutions for a few examples of these equations are known.

The generalized Hypergeometric functions are shown to be the asymtotic solutions of a simple difererential equation in a systematic way. New identities for the absolute values

of these functions are derived. The generality of the results obtained here induces us to consider the utility of defining Hypergeometric functions of matrix parameters ${ }_{1} F_{1}(A, B ; z)$ with z complex, similar extensions exist already: generalizations with complex parameters but matrix argument or parameters and argument defined in arbitrary finite fields for example. Let's note finally that making the change

$$
y=\exp (-t)
$$

our system becomes of the form

$$
\partial_{y} x=\left(\frac{A}{y}+B\right) x
$$

so we have found also a solution for this system, the Hypergeometric functions appear now as regular solutions for $y \rightarrow 0$.

\section{A Appendix: some formulas about Hypergeometric Func- tions}

See [12] and references therein for all of these definitions and formulas. The Confluent Hypergeometric function is defined by

$$
{ }_{1} F_{1}(a, b ; z)=\sum_{n=0}^{\infty} \frac{(a)_{(n)}}{(b)_{(n)}} \frac{z^{n}}{n !}
$$

where the Pochammer symbol is

$$
(z)_{(n)}=\Gamma(n+z) / \Gamma(z)
$$

The generalized Hypergeometric function is defined by

$$
{ }_{p} F_{q}\left(a_{1}, \ldots, a_{p}, b_{1}, \ldots, b_{q} ; z\right)=\sum_{n=0}^{\infty} \frac{\left(a_{1}\right)_{(n)} \ldots\left(a_{p}\right)_{(n)}}{\left(b_{1}\right)_{(n)} \ldots\left(b_{q}\right)_{(n)}} \frac{z^{n}}{n !}
$$

The integral 


$$
\int_{0}^{t} x^{\gamma-1}(t-x)^{c-\gamma-1}{ }_{1} F_{1}(a, \gamma ; x) d x=t^{c-1} \frac{\Gamma(\gamma) \Gamma(c-\gamma)}{\Gamma(c)}{ }_{1} F_{1}(a, c ; t) ; \quad[\Re c, \gamma>0]
$$

is a special case of

$$
\begin{gathered}
\int_{0}^{1}(1-x)^{(\mu-1)} x^{b_{1}-1}{ }_{p} F_{q}\left(a_{1}, \ldots, a_{p} ; b_{1}, \ldots, b_{p} ; a x\right) d x= \\
\frac{\Gamma(\mu) \Gamma\left(b_{1}\right)}{\Gamma\left(\mu+b_{1}\right)}{ } F_{q}\left(a_{1}, \ldots, a_{p} ; \mu+b_{1}, b_{2}, \ldots, b_{q} ; a\right) ; \\
\quad\left(\Re\left(\mu, b_{1}\right)>0, p<q+1\right)
\end{gathered}
$$

Some other important formulas are:

$$
\begin{aligned}
\frac{d_{1} F_{1}(\alpha, \gamma ; z)}{d z} & =\frac{\alpha}{\gamma}{ }_{1} F_{1}(1+\alpha, 1+\gamma ; z) \\
{ }_{1} F_{1}(\alpha, \gamma ; z) & =\exp z{ }_{1} F_{1}(\gamma-\alpha, \gamma ;-z) \\
\frac{z}{\gamma}{ }_{1} F_{1}(\alpha+1, \gamma+1 ; z) & ={ }_{1} F_{1}(1+\alpha, \gamma ; z)-{ }_{1} F_{1}(\alpha, \gamma ; z) \\
\alpha_{1} F_{1}(\alpha+1, \gamma+1 ; z) & =(\alpha-\gamma){ }_{1} F_{1}(\alpha, \gamma+1 ; z)+\gamma{ }_{1} F_{1}(\alpha, \gamma ; z) \\
\alpha_{1} F_{1}(\alpha+1, \gamma ; z) & =(z+2 \alpha-\gamma){ }_{1} F_{1}(\alpha, \gamma ; z)+(\gamma-\alpha){ }_{1} F_{1}(\alpha-1, \gamma ; z)
\end{aligned}
$$

From [16] we know that the real zeros $x_{j}$ of ${ }_{1} F_{1}(a, c ; x)$ for a,c real satisfy the bounds

$$
(c-2 a)-2 \sqrt{ }(a(a-c)-c)<x_{j}<(c-2 a)+2 \sqrt{ }(a(a-c)-c)
$$

The smallest real zero $x_{1}=x_{\text {min }}$ satisfy

$$
x_{\min }<\frac{c(c+2)}{c-2 a}
$$

Appliying the same bound to the expression (74) we deduce the lower bound for the maximal real zero $x_{\max }$

$$
\left(x_{\min }<\right) \frac{c(c+2)}{c-2 a}<x_{\max }
$$

\section{Acknowledgements.}

I would like to thank to Peter Minkowski for many enlighthening discussions. This work has been supported in part by the Wolffman-Nageli Foundation (Switzerland) and by the MEC-CYCIT (Spain). 


\section{References}

[1] T.Kuo,J.Pantaleone. Phys. Rev. Lett. 57,14 (1986) p.1805. PURD-TH-89-3 (1989).

[2] S.P. Mikheyev, A. Smirnov. Phys. Lett. B. Vol.200,4 (1988) p.560. Il Nuovo Cimento.. Vol.9 (1986) p.17.

[3] S. T. Petcov. Phys. Lett. B vol.214,2 (1988) p.259.

[4] J.N. Bahcall, R. Ulrich. Rev. Mod. Phys. 60 (1988) 267.

[5] C. Zener. Proc. Royal Soc. London. Ser A,137 (1932) p.696.

L. Landau. Phys. Z. Soviet Union 2-46 (1932).

[6] S. T. Petcov. Phys. Lett. B Vol.191 (1987) p.299.

[7] W.C. Haxton, Phys. Rev. D 35 (1987) 2352.

[8] D. Notzold, MPI-PAE/Pth 08/87 (Munich 87).

[9] S. T. Petcov. Phys. Lett. B vol.200,3 (1988) p.373.

[10] U. Toshev. Phys. Lett. B196 (1987) 170.

[11] A. Abada, S.T. Petcov. Phys. Lett. B Vol.214,2 (1988) p.139. Phys. Lett. B vol.279 (1992) p.153.

[12] I.S. Gradshteyn, I.M.Ryzhik. Table of Integrals,series and products. Academic Press.1980.

[13] R.B.Dingle. Asymtotic Expansions. Academic Press.1973.

[14] T.Kuo,J.Pantaleone. Phys. Rev. D35,11 (1987) p.3432.

[15] P.W. Anderson. "Absence of Diffusion in Certain Random Lattices". Phys. Rev. 1095, 1492-1507 (1958).

[16] S. Ahmed J. of Approximation Theory 34, 335-347 (1982). 

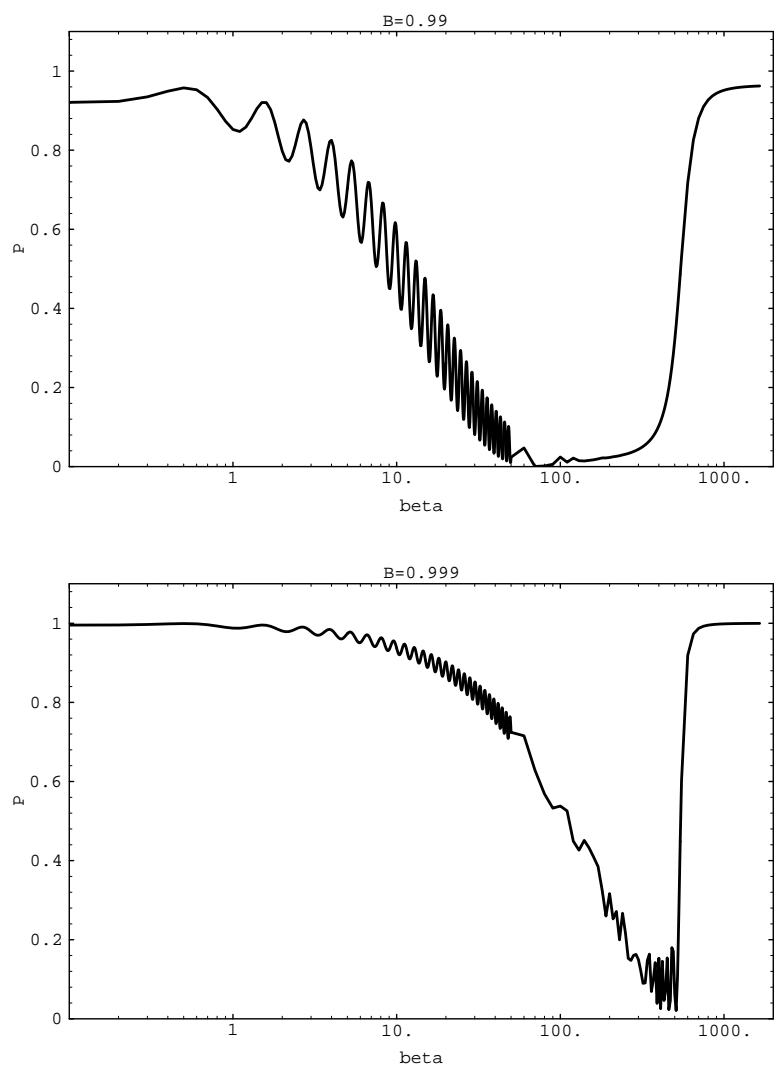

Figure 1: The function $\left|{ }_{1} F_{1}(B i \beta, i \beta,-i 550.2)\right|^{2}$ for two different B's near 1 , as a function of $\beta$. This corresponds approximately to the oscillation probability for a neutrino produced at $r / r_{0}=0.25$ as function of $\beta=\Delta m^{2} / 2 E \lambda$. 

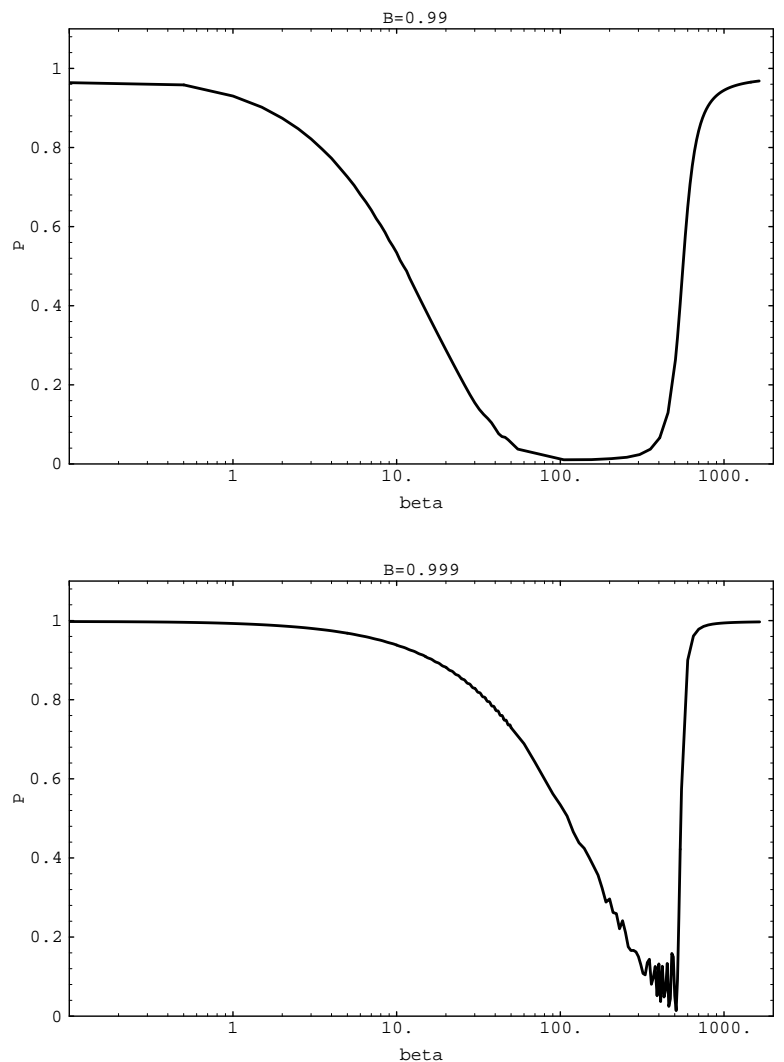

Figure 2: $P_{e e}$ (two neutrino species, Formula 36) for a neutrino produced at $r / r_{0}=0.25$ as a function of $\beta=\Delta m^{2} / 2 E \lambda$ and two different mixing angles: $B\left(=\cos ^{2} \theta\right)=0.99,0.999$. 

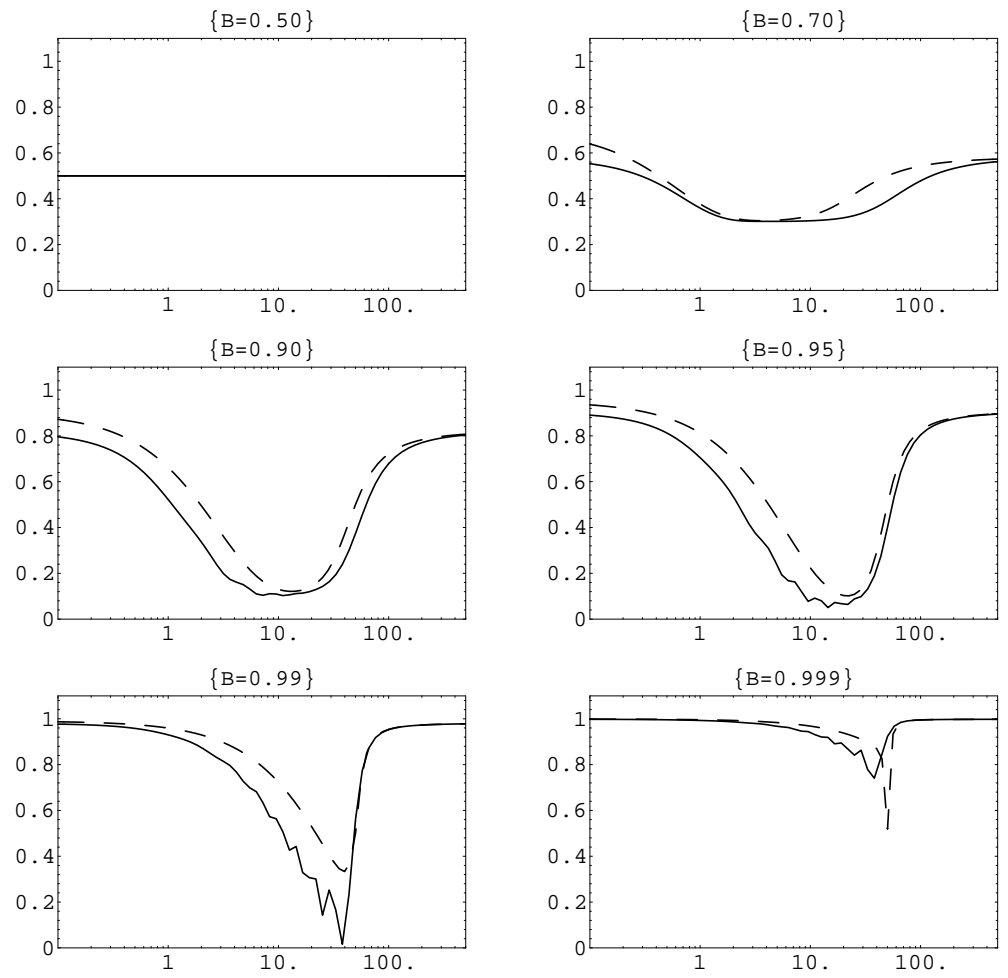

Figure 3: As in Figure (2) but for a neutrino created near the surface $(|z| \approx 20)$ as a function of the mixing angle. The dashed line corresponds to the Parker approximate solution (as it is given by Formula 2.18 in [14]). 

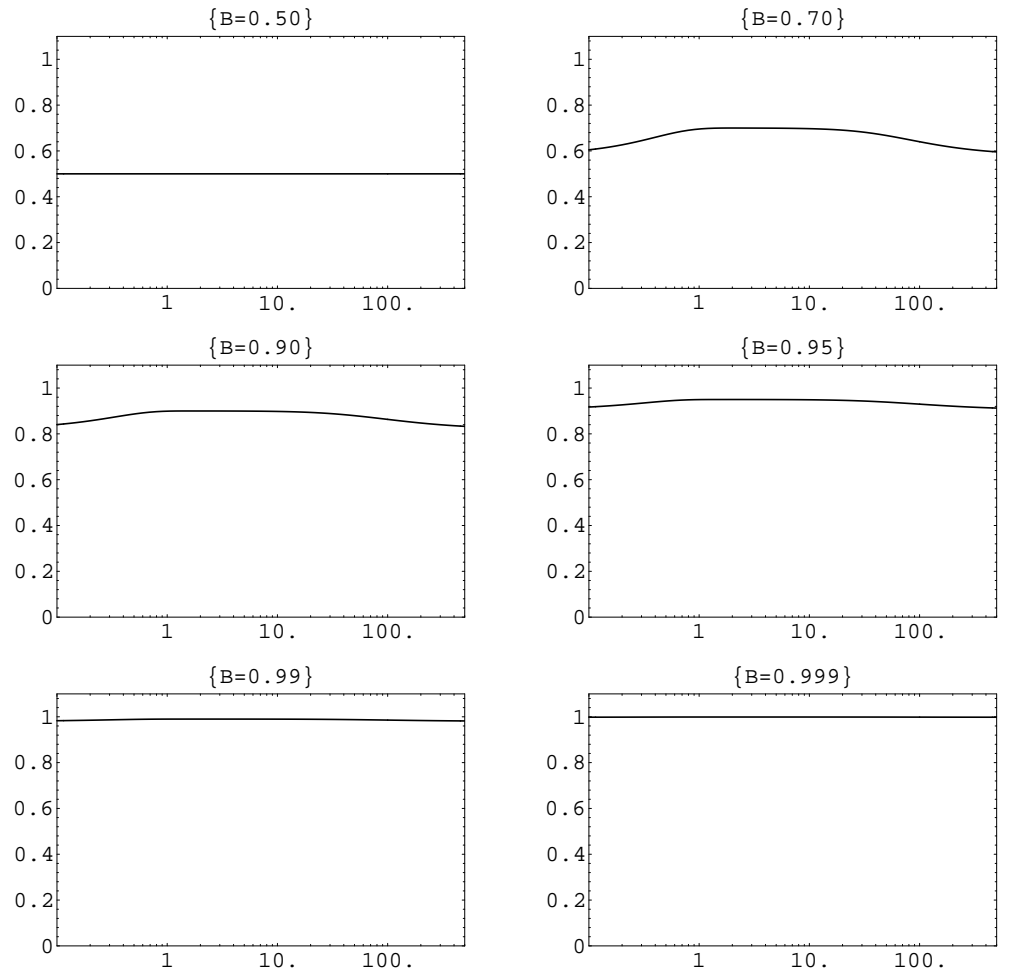

Figure 4: As Fig. (3) but for antineutrinos (reverse sign in the argument $z$ of the hypergeometric function).In this case there is not resonance (it is possible to distinguish some "anti"-resonace behaviour for larger mxing angles). 


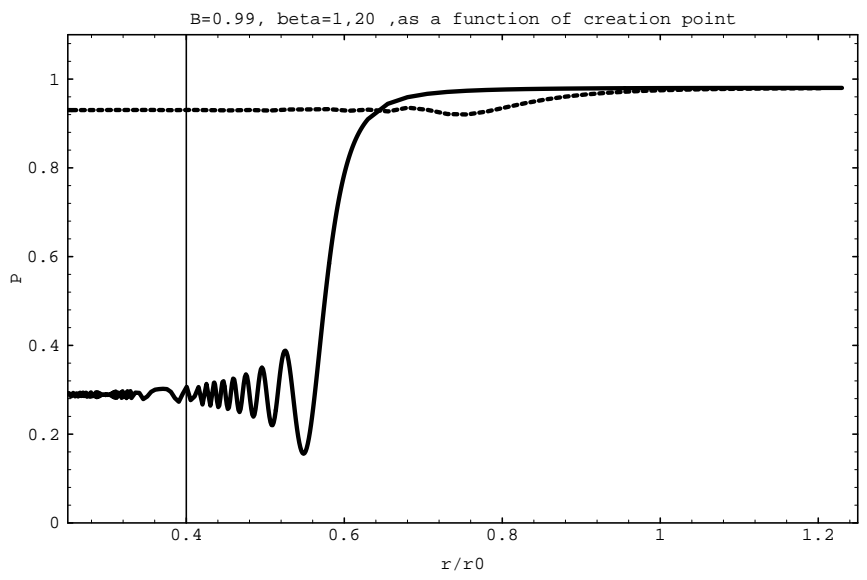

Figure 5: $\quad P_{e e}$ (Eq. (36)), as a function of the creation point (in solar radius fraction) for a fixed mixing angle $\cos ^{2} \theta=0.99$ and two different $\beta=\Delta m^{2} / 2 E \lambda=1,20$ (respectively upper and lower curves ). 


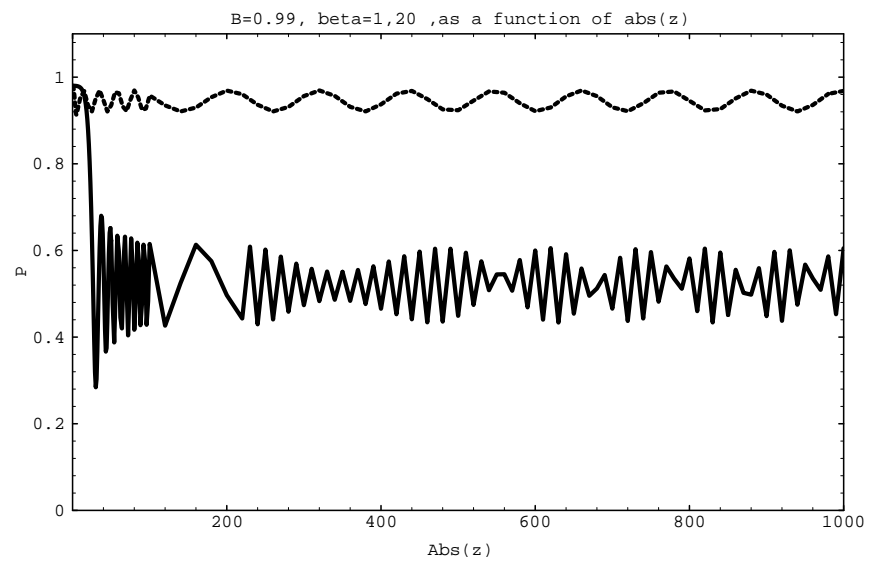

Figure 6: Function $\left|{ }_{1} F_{1}(i B \beta, i \beta, z)\right|^{2}$ as a function of $|z|$, for different values of the parameters. $\mathrm{B}=0.99$, the upper and lower curves correspond respectively to $\beta=1$ and 20 . 